Journal of Computer Science 4 (1): 42-50, 2008

ISSN 1549-3636

(C) 2008 Science Publications

\title{
Dynamic Head Cluster Election Algorithm for Clustered Ad-Hoc Networks
}

\author{
${ }^{1}$ Arwa Zabian, ${ }^{2}$ Ahmed Ibrahim and ${ }^{3}$ Fadi Al-Kalani \\ ${ }^{1}$ Department of CIS \\ ${ }^{2}$ Department of CS \\ ${ }^{3}$ Department of Computer Education, Irbid National University, Irbid, Jordan
}

\begin{abstract}
In distributed system, the concept of clustering consists on dividing the geographical area covered by a set of nodes into small zones. In mobile network, the clustering mechanism varied due to the mobility of the nodes any time in any direction. That causes the partitioning of the network or the joining of nodes. Several existing centralized or globalized algorithm have been proposed for clustering technique, in a manner that no one node becomes isolated and no cluster becomes overloaded. A particular node called head cluster or leader is elected, has the role to organize the distribution of nodes in clusters. We propose a distributed clustering and leader election mechanism for Ad-Hoc mobile networks, in which the leader is a mobile node. Our results show that, in the case of leader mobility the time needed to elect a new leader is smaller than the time needed a significant topological change in the network is happens.
\end{abstract}

Key words: Dynamic head cluster, Ad-Hoc networks, mechanism, leader, election algorithm

\section{INTRODUCTION}

Mobile network is a network in which the location of the nodes is variant with the time. The mobile networks can be configured in two manners, infrastructure network and infrastructureless network. In infrastructure network a fixed node called base station with unlimited power supply is responsible of the communication between the mobile nodes in the network. In infrastructureless network all the nodes are free to move in any direction without any preexisting fixed infrastructure, all the nodes has limited battery life and all the nodes can communicate to each other within the transmission range. In such network, the mobile hosts must cooperate to each other forwarding packets to maintain the network connectivity and to benefits of the shared resources in the network. For that, a routing algorithm is needed to organize the forwarding of packets in the network. There are many routing algorithms proposed for mobile network ${ }^{[1-6]}$.

Ad-hoc wireless network is characterized by dynamic topology, multihop communication and high power consumption. So, the frequent change in the network topology can vary the distribution of the nodes in the network, where nodes can aggregate in a single connected components (cluster) doing merge operation, or can be distributed in different components (split), for that it is necessary a technique that maintains a good balance of the node distribution in the network.

Clustering is a well known technique for grouping nodes that are close to each other in the network and to reduce the useful energy consumption. Energy consumption in mobile network can be either useful or wasteful. Useful energy consumption can be in receiving or transmitting data, processing request or forwarding data/request to neighboring nodes.

The concept of clustering consists on dividing the geographical area covered by the mobile nodes into small zones and then select in each cluster a node called head cluster is responsible for coordinating the communication with the nodes of the other clusters (intra-cluster) and the communication with the mobile nodes in the same cluster (inter-cluster). The head cluster elected must hold determined characteristics such as longer battery life and minimum average distance to a large subset of the node of the cluster. Designing a cooperative real time control system requires a good understanding of the system and the way in which cooperates to achieve the common goal that is, the maintenance of the system connectivity and the system life time as long as possible. For that, the problem of choosing a dynamic coordinator can be reduced to the leader election problem. 
Leader election problem is a very important problem in wired and mobile network. But, the existing solution for leader election problem proposed for distributed system cannot be applied in wireless Ad-hoc network where the topology change frequently and nodes, links fail.

In this study, we propose a dynamic head cluster algorithm for clustered ad-hoc network. The system is a mobile network organized in clusters in which the head cluster is mobile node that can move in all directions. That means, the head cluster in one cluster after an Interval of time may becomes a mobile host in another cluster. In such case, our algorithm allows the election of another head cluster; in a manner that no one clusters still without leader (the terms leader and head cluster will be used interchangeably) and each cluster has only unique head cluster.

Problem definition: The leader election problem in general is the problem of electing a unique leader from a fixed set of nodes. In mobile network this problem will change because all the nodes are mobile in addition to the mobility of the leader it self. That means, the leader can be leader in one cluster and after an interval of time it moves to another area in which it becomes a mobile terminals. However, in mobile networks the leader election algorithm must be dynamic, that means must tolerate the topology change and will terminate with the election of unique leader in a manner to avoid the situation in which the system is without leader as less as possible.

Electing a leader in clustered mobile network is important because a coordination mechanism for the communication is needed to allow the connectivity in the network (no isolated nodes, no route broken) as long as possible.

The problem studied in this study, is given a graph $\mathrm{G}=(\mathrm{V}, \mathrm{E})$, we will define a mechanism to organize the nodes of $\mathrm{G}$ in clusters given the transmission range and electing a head cluster that holds determined parameters like its closeness to many nodes in the cluster (called neighbors) and to have the higher battery life between all of its neighbors. The election process will start randomly without any supervisor.

\section{DYNAMIC HEAD CLUSTER ELECTION ALGORITHM (DHCEA)}

System description: Our system as any network model is represented by a graph $\mathrm{G}=(\mathrm{V}, \mathrm{E})$ where $\mathrm{V}$ is a set of mobile nodes $|\mathrm{V}|=\mathrm{n}$ and $\mathrm{E}$ is a bidirectional links. The nodes are organized in clusters given the transmission range. That means, the nodes in the same cluster are in the transmission range of the root node, they can communicate by a way of the root or directly to each other if they are in the transmission range of each other. Where $\mathrm{i}, \mathrm{j} \in \mathrm{V} \Rightarrow \mathrm{a}$ link between $\mathrm{i}, \mathrm{j}$ exists if and only if $\left|a_{i}-a_{j}\right|<r$ where $a_{i}, a_{j}$ are the location of the nodes $i, j$ and $r$ is the transmission range. Each cluster has a node called Head Cluster (HC) chosen given a determined parameters. The $\mathrm{HC}$ is a mobile node that can change its location given the time. The role of the $\mathrm{HC}$ is topology control and to carry out a communications between the nodes of the cluster and other nodes in the network that are out of range and to organize the communication between the nodes of the cluster to maximize the system life time. For that, the $\mathrm{HC}$ has to collect information about the nodes of its cluster and assigns to each node an identity used for the communication in the network. So, the HC periodically exchange an acknowledgement messages with the nodes of the cluster. The information exchanged is the identity of the node and its power level. The HCs that are in the transmission range to each other are connected and work as intermediate nodes maintaining the connectivity between all the nodes of the network. The other nodes in the cluster are mobile terminals (MT) have the freedom to move in any direction with pause point given random way mobility models where in each time slot the location of a node is observed given a reference point group mobility model (HC).

\section{Assumptions:}

- All the nodes involved in our system are in the active state. A down node is considered out of the range and when this node resumed activity will be considered as new node enter the cluster

- All the nodes in the network are similar in the power consumption and transmission range

- We speak about completely dynamic system that means all the nodes are mobile and without any additional power supply. The battery power is reduced with the time (the other possibility that a node may stay a fixed interval of time in a location in which can recharge its battery is out of our interest in this study)

- We assume that the topology of the network does not change during the interval of $\mathrm{HC}$ election that implies the time of leader election algorithm must be small as possible

Algorithm description: Before describing the algorithm it is useful to define some notations will be used in the algorithm description: MT refers to a mobile 
terminal, $\mathrm{HC}$ refers to the Head cluster, $\mathrm{P}(\mathrm{HC})$ refers to the power of the $\mathrm{HC}$ and $\mathrm{D}(\mathrm{HC})$ refers to the number of neighbors of the HC. N represents the number of nodes in the network.

In constructing our network the DHCEA uses three type of messages are: Leader Election message (LE) that is sent in the network when the nodes start working or when the leader is moved to another area. The second type of messages is the Neighbor Discovering message (ND) that is sent by the $\mathrm{HC}$ or any discovered node in the cluster formation phase to collect information about the nodes in its environment. The last type of messages is the Acknowledgement message (ACK) will sent periodically by the $\mathrm{HC}$ to maintain information about the network.

The algorithm runs in three phases are: the Head Cluster Election Phase and the Cluster Formation Phase and finally, the Network Maintenance Phase.

\section{Phase 1: Head cluster election:}

- A node $\mathrm{U}$ chosen randomly starts discovering its environment by broadcasting a leader election message (LE) to which is attached its power level and its identity and sets a time out $t$. $t$ is the time needed to a radio waves to travel in an environment without obstacles a distance equal to the maximum area covered by the transmission range $(t=21+\varepsilon$; where 1 is the maximum distance covered by the transmission range and $\varepsilon$ is the processing time at the destination will be ignored) and $\mathrm{r}=1 \mathrm{~d}^{\alpha}$. Each node hears the leader election message and wants to join the cluster; it must send a response message that contains its power level and its identity. If $U$ does not receive any response, it waits a time $t$ and sends another LE message until the reception of a response or until hearing a LE message comes from other nodes

- If two nodes start the leader election process at the same time and the two nodes can hear each other, one stopped and the other continues the election process. If the two nodes cannot hear each other, each node elects a leader in its environment and at the end of the process is chosen only one as leader (step 6, phase1).

- All the response messages received is inserted in a queue, from which $U$ sorts one node with higher power level as leader

- If there are two nodes in the queue with the same power level (higher level) is chosen that with higher number of neighbors

- Then $U$ distributes the leader identity in the cluster
- If there are another leader in the same cluster (for scalability issue we assume that not all the nodes in the cluster are reachable in only one hop). The two headers will be compared given the higher power level and given the proximity to more nodes in the cluster (a header is supposed to be close to a large part of the nodes of the cluster). If the two HCs have the same power level, is chosen the head cluster with large set of neighbors. Otherwise, one $\mathrm{HC}$ is chosen as $\mathrm{HC}$ and the other will be considered as substitute $\mathrm{HC}$ (considering that the $\mathrm{HC}$ is a mobile node and can change location with the time). The power level $\mathrm{P}$ varies from 1-k, where $\mathrm{k}$ is the maximum power.The unreachable node by the header can be reached by multihops given another MT). Figure 1 shows the flowchart that describes the first phase of the algorithm that is head cluster election

Phase 2: Cluster formation phase: In this phase:

- The elected HC broadcast a ND message to construct its cluster, all the nodes that hear the ND message and they want to join it, they must send a response message. Based on which the $\mathrm{HC}$ assigns to them their identity in the network

- The ND message can be heard by two types of nodes: nodes not yet discovered (new entered this area, or were down and resumed activity) that means haven't a parent. If it wants to join to this $\mathrm{HC}$ it must send a response message. The second

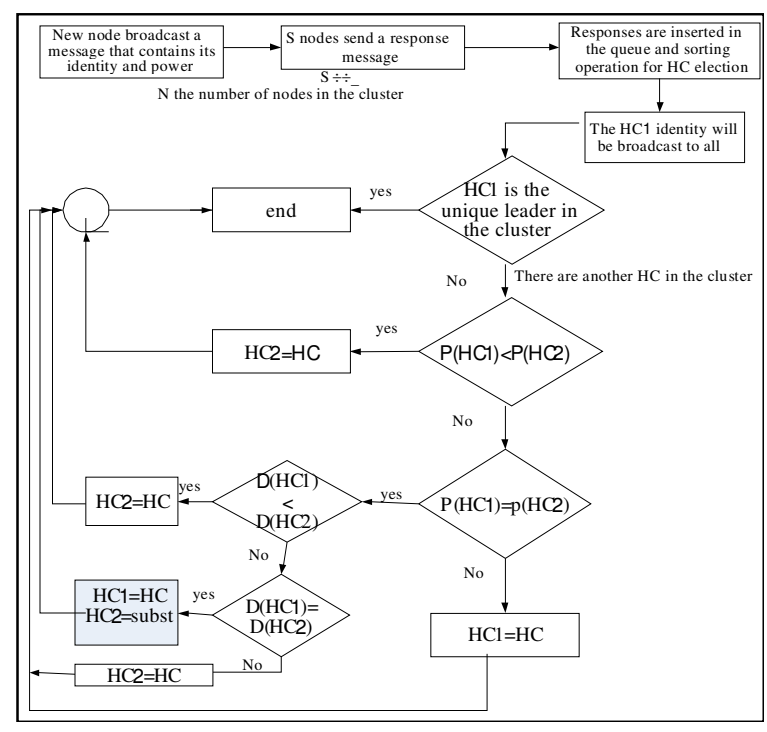

Fig. 1: Head cluster election 
type of nodes is nodes have been discovered and has its parent that means belong to another cluster This type of nodes represent an important nodes in the routing process because it represents an intermediate nodes, such node has the liberty to take this link as additional link or to ignore the ND message.

- Based on the responses received the HC constructs its routing table in which is inserted the identity of the nodes connected to it and its power level (from the same cluster or from the other clusters). And will be denoted as children to which is assigned their identities

- Steps 1, 3 are repeated in each cluster until the construction of all the network

- If a HC node hears the ND message comes from a MT or from another $\mathrm{HC}$ and it wants to collaborate, it must send a response message. In the case where the ND messages were exchanged between a HC's, each $\mathrm{HC}$ add in its routing table a new row in which is inserted all the HC's connected to it (that means are in its transmission range)

- If a node (MT) receives a ND message from two HC's, it can choose only one as $\mathrm{HC}$ from which it takes its identity and the other $\mathrm{HC}$ will be reserved as backup links. Such nodes represent a high importance in our system because it will be considered as intermediate nodes that can carry out a communication between two clusters with low cost. For example, in Fig. 2, both nodes 011 and the node 043 represent an intermediate node between two clusters are consecutively G1 andG2, G4 and G3

The address assignment: One of the roles assigned to the $\mathrm{HC}$ is to assign a unique identity to the nodes of its cluster. The addresses assigned are composed on three

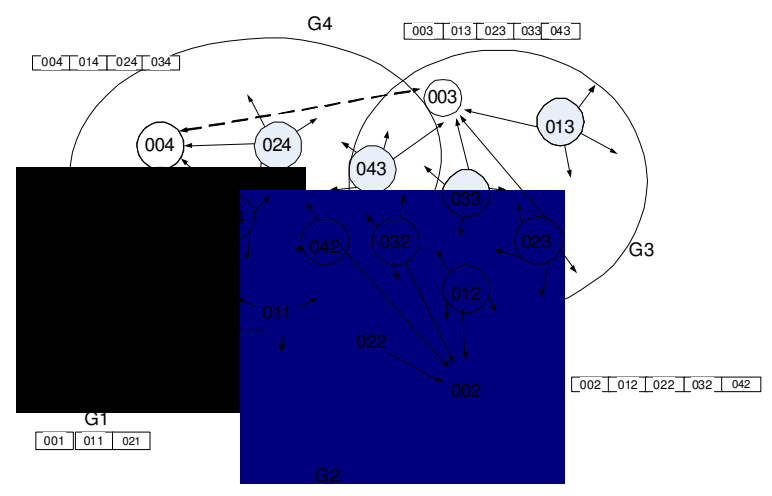

Fig. 2: Phase 2, cluster formation phase components are: $\mathrm{XYZ}$ where each one can be 4 bits are necessary to represent the numbers from $0-9$. $Z$ is conserved to denote the HC identity, where it corresponds to the cluster number, it takes values from $1-9$, for example the identity of the HC of G1, will be 001 and all the nodes of the cluster must carry the HC's address. $\mathrm{Y}$ is a number assigned by the $\mathrm{HC}$ it identifies the node; it varies from 1 to 9 . For example the MT's of the cluster $\mathrm{G}_{1}$ must have the identity $0 \mathrm{Y} 1$. $\mathrm{X}$ is reserved for scalability issues, if the number of nodes in the cluster is increased (more than 9), a new identities can be assigned to the new nodes where we can extend the number of nodes in each cluster to $2^{9}$ without any duplication address. $\mathrm{X}$ is assigned given the vicinity to well defined node in the cluster. That means, if a new node enters to the cluster and all the identities of the form $0 \mathrm{YZ}$ are occupied the identity of the new node will be defined given the nearest node to it. The address assignment is explained in the example (1).

Phase 3: Network maintenance: In this phase the network maintenance is done, where the HC sends periodically an acknowledgement messages to collect information about its cluster. If some change is happened in the network topology, the change will be attached in biggyback to the acknowledgement message, reducing in that the flooding of the messages in the networks. The information exchanged between the HC and the MT's or between the MT's are: the battery level and the change in the routing table.

Mobility management: We speak about Ad-Hoc mobile system where all the nodes can move and change its location with the time and in particular the $\mathrm{HC}$. When the HC

still to leave its cluster, it must send a notification message to all its children and here we can find the following two cases:

- In the cluster has been elected only one $\mathrm{HC}$ without substitute and the Head Cluster Election phase must be restarted

- In the cluster a substitute $\mathrm{HC}$ has been elected and it will become a HC. That is explained in flowchart in Fig. 1

Example (1): Figure 3A, B, C and D show a simulation of the first and second phases of DHCEA in a graph of 11 nodes. Figure 3A simulates the leader election phase where two nodes randomly start broadcasting a LE message. In part A, from Fig. 3B, the nodes that hear LE message and want to cooperate send a response message. Part B in Fig. 3B represents the formation of 


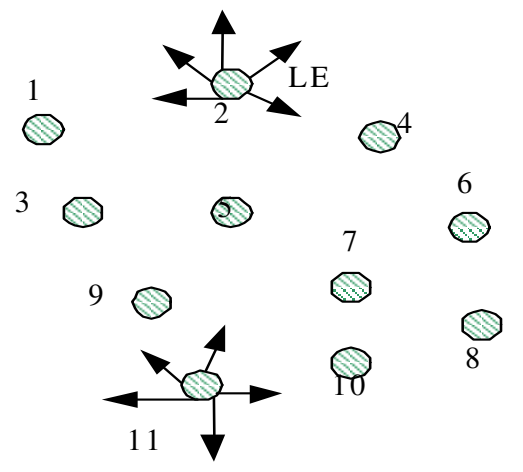

Fig. 3A: Leader election phase

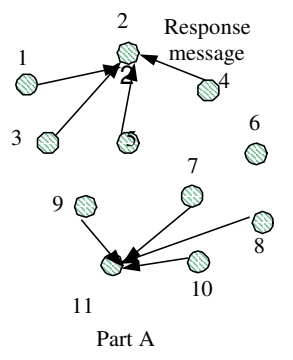

Group formation

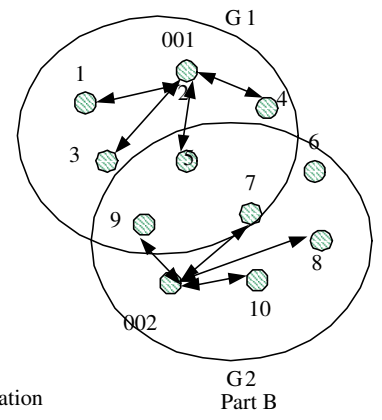

Fig. 3B: Cluster formation
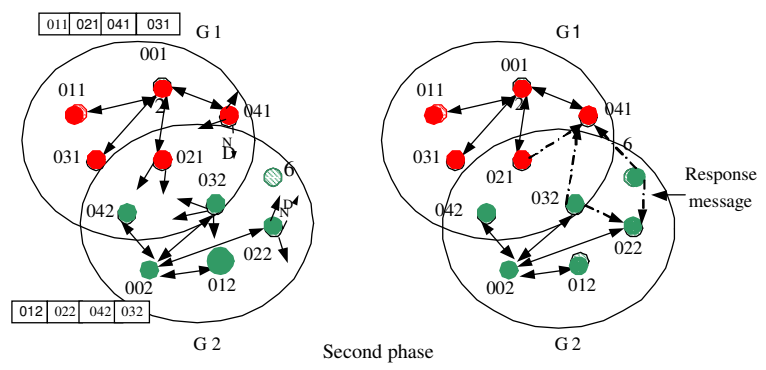

Fig. 3C: Address assignment

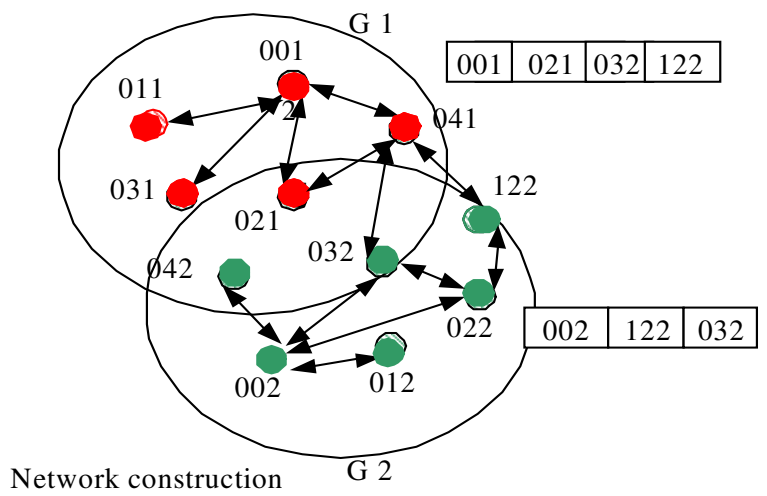

Fig. 3D: Network construction the The right part of Fig. 3C represents how the leaders elected assign the identities to their child and each one constructs its routing table. Then, each discovered node represented by bold point starts discovering its environment.

Algorithm analysis: Our algorithm is proposed for dynamic leader election and cluster formation in ad-hoc wireless networks. Our algorithm ensures that all the time each cluster has a leader and only one leader (from the algorithm construction and mobility management).

Number of messages exchanged for cluster formation: In the cluster formation phase each discovered node broadcasts only one Neighbor Discovering message to collect information about the node to which is connected directly that means the number of ND message is at most N. In addition, each node want to collaborate must send a response message.

So, the number of response messages is at most $\mathrm{d}$ $<\mathrm{N}$. For that the number of messages exchanged is: $\mathrm{N}+\sum \mathrm{d}<<2 \mathrm{~N}$ that means is linear with $\mathrm{N}$.

The main advantage of our algorithm is that the clusters are fixed. That means, when the network is constructed and the clusters are formed the nodes can move, enter or exit the cluster but all the time the nodes that are in the same area defined by the cluster must have a leader elected from the nodes that are in the same area.

\section{RESULTS AND RESULTS ANALYSIS}

To study the performance of our proposed algorithm in organizing a network in a set of clusters. And electing in each cluster a unique node as leader in a manner that no cluster is without leader in spite the leader mobility.

We modeled the system as a set of nodes organized in clusters; using Delphi program where we have been prepared a script that simulate a set of nodes organized in a set of clusters and then the leader election process is done given the flowchart presented in Fig. 1. Each node in our system is described by the following two variables :

- $\quad$ P the power of the node that is randomly assigned and takes values from 20-100. Where 20 is considered the minimum power needed to a node to carry out a communication. And 100 is considered full power, is assumed that the leader must have full power

- D the number of neighbors refers to the number of nodes in each cluster. When our algorithm (DHCEA) run in a graph $G$ each node must be connected to a set of nodes $\mathrm{S}$ where $\mathrm{S} \subseteq \mathrm{d}$ 
Table1: The variation of the time needed for leader lection $(\tau)$ with the network size $(\mathrm{d}=20)$

\begin{tabular}{lll}
\hline Number of nodes $(\mathrm{N})$ & $\tau(\mathrm{ms})$ & Cluster number \\
\hline 100 & 39 & 4 \\
150 & 54.5 & 5 \\
200 & 70 & 7 \\
250 & 86 & 9 \\
300 & 86 & 10 \\
350 & 102 & 12 \\
400 & 117 & 14 \\
450 & 133 & 15 \\
\hline
\end{tabular}

Table2: The variation of the time needed for leader lection $(\tau)$ with the network size $(\mathrm{d}=25)$

\begin{tabular}{lll}
\hline Number of nodes $(\mathrm{N})$ & $\tau(\mathrm{ms})$ & Cluster number \\
\hline 100 & 39 & 5 \\
150 & 54 & 8 \\
200 & 55 & 10 \\
250 & 70 & 13 \\
300 & 86 & 15 \\
350 & 102 & 17 \\
400 & 117 & 20 \\
450 & 133 & 23 \\
\hline
\end{tabular}

In our system $\mathrm{N}$ represents the number of nodes in the network ( that is shown in snapshot 1 Fig. 6). We are interested in calculating the time needed to elect a leader to define the behavior of the system in the case of leader mobility. Different simulations have done varying the number of nodes $\mathrm{N}$ and varying the cluster size d. In each time, the leader as the node with higher $\mathrm{P}$ and higher number of neighbors $\mathrm{S}$ is elected.

As assumed in our algorithm, the network topology does not change during the leader election process for that in our simulation we have considered the network fixed and we will calculate $\tau$ the time needed for a leader election to predict the behavior of the network in the case of leader mobility.

The variation of $\tau$ with $\mathbf{N}$ (Scalability): To study the variation of the time election $\tau$ with the network size we have done many simulations in which we fixed the number of nodes $d$ in each cluster $(20,25)$ varying the network size each time from 100 to 450 (Table 1 nad 2, respectively). The results presented in Table 1 and 2 show that $\tau$ is increased linearly with the network size but the time is still independent on the cluster number. Comparing lines 3, 8 in Table 1 and 2, we note that for a fixed number of network the time needed for leader election is independent on the cluster size. In addition, the time variation in different network size $(\mathrm{N}, \mathrm{N}+50)$ is with fixed interval $\Delta \tau=\tau_{2}-\tau_{1} \approx 16 \mathrm{~ms}$.

The variation of $\tau$ with the cluster size (d): To study the effect of cluster size in the variation of $\tau$, we have
Table 3: The variation of the time needed for leader lection $(\tau)$ with the cluster size in a network of 100

\begin{tabular}{ll}
\hline Cluster size $(\mathrm{d})$ & $\tau(\mathrm{ms})$ \\
\hline 10 & 39 \\
20 & 39 \\
30 & 39 \\
40 & 39 \\
50 & 39 \\
60 & 39 \\
& \\
\hline
\end{tabular}

Fig. 4: The variation of the time needed for leader election $(\tau)$ with the network size

\begin{tabular}{ll}
\hline Cluster size $(\mathrm{d})$ & $\tau(\mathrm{ms})$ \\
\hline 10 & 39 \\
20 & 39 \\
30 & 39 \\
40 & 39 \\
50 & 39 \\
60 & 39 \\
70 & 39 \\
\hline
\end{tabular}

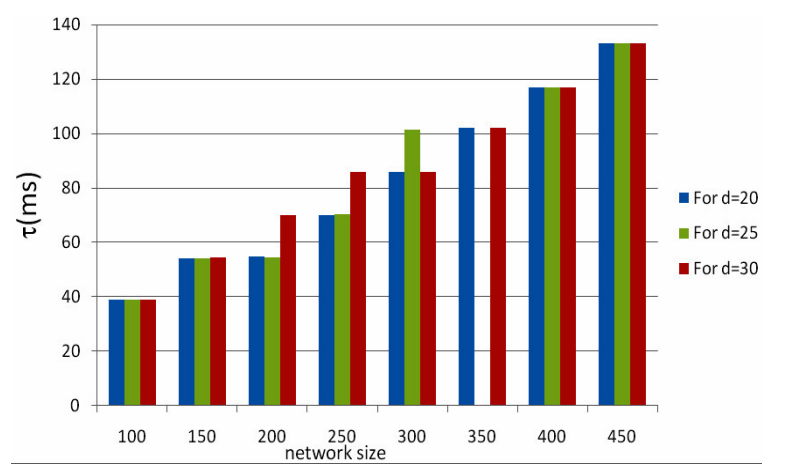

Fig. 4: The variation of the time needed for leader election $(\tau)$ with the network size

varied $\mathrm{d}$ in a fixed network size (100 nodes) (Table 3). Table 4 shows that $\tau$ is independent on the cluster size.

Results analysis: Analyzing the results presented in Table 1 and 2, we can see that the time needed for leader election process $(\tau)$ is independent on the cluster size (Fig. 4) and on the number of neighbors (Fig. 5), but it depends only on the total number of nodes in the network N. So, given our results the time needed $(\tau)$ can be represented by the following mathematical formula:

$$
\tau= \begin{cases}\mathrm{N} / 50 * \Delta \mathrm{t} ; & 50 \leq \mathrm{N} \leq 500 \\ \mathrm{~N} / 50 / \Delta \mathrm{t} \mp \varepsilon ; & 500 \leq \mathrm{N} \geq 850\end{cases}
$$

$\varepsilon$ is a time represents the differences between the mathematical calculation and the simulation results $4 \leq \varepsilon \leq 20$ (ms). In Fig. 6 is noted that $\tau$ vary between an 


\section{the variation of the time with the cluster size}

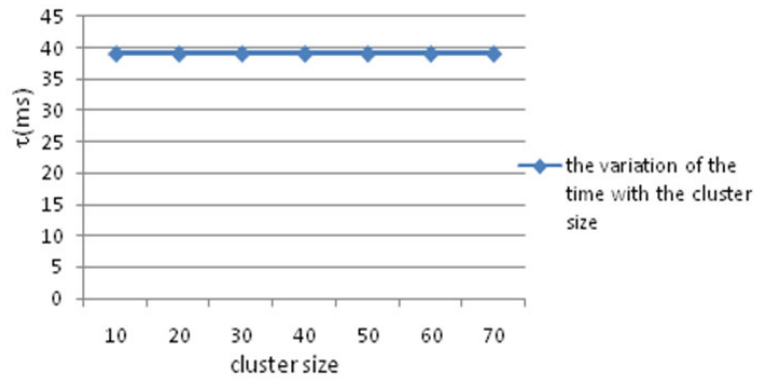

Fig. 5: The variation of the time needed for leader election $(\tau)$ with the cluster size

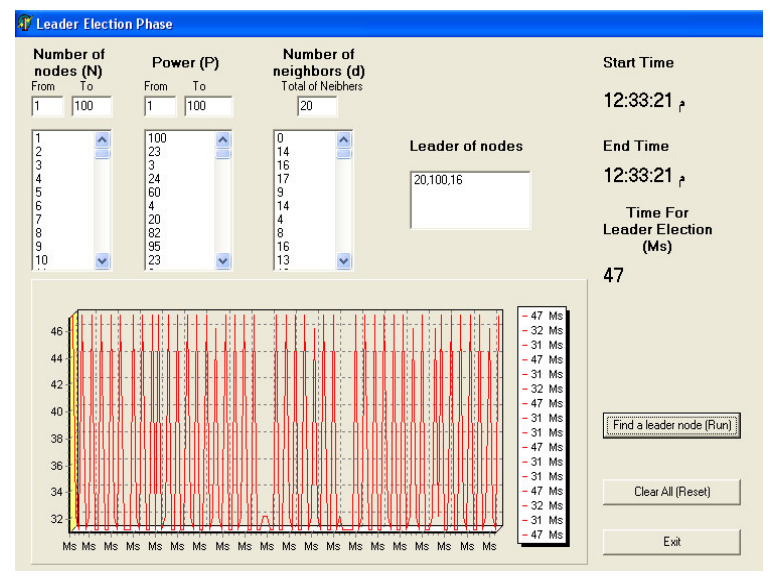

Fig. 6: Snapshot 1

interval $\Delta \tau$ (in snapshot (1) $\Delta \tau=47-31 \cong 16 \mathrm{~ms})$. From Table 1 and 2, we can see that the maximum value of $\tau$ is $133 \mathrm{~ms}$. Considering that in all mobile systems, any mobile node can move with a velocity of maximum $250 \mathrm{~km} \mathrm{~h}^{-1}$ and the transmission range can vary from $(200 \mathrm{~m}-35 \mathrm{~km})$.So, a mobile node needs a time from (2.88-504 s) respectively to leave its area. Based on that we can conclude that in our system the time needed to elect a new leader is small than the time needed to a mobile node to leave a given area. That means, no cluster is stay without a leader and if the leader is moved out of range the process of leader election will not influences in the system performance because it is done in a small time in which the topology of the network cannot change widely (that confirms our assumption in section 3).

\section{CONCLUSION AND FUTURE WORKS}

Clustering is a well known technique for grouping nodes that are close to each another in the network.
In clustering system, any node can become a cluster head if it has the necessary functionality.

A node registers with the nearest cluster head and becomes a member of that cluster.

Clusters may change dynamically reflecting the mobility of the underlying network. $\mathrm{In}^{[7]}$, is proposed an energy efficient clustering technique based on the weight cost function. In this technique two main parameters are used to the cluster construction and to the head cluster election are: the transmission power levels and the threshold of the number of neighbors $(\sigma)$ to be covered.

A weight cost function used in the head cluster election includes three parameters are: power level $\mathrm{W}_{1}$ and the residual power $\mathrm{W}_{2}$ and the speed $\mathrm{W}_{3}$. Where the head cluster is elected with lower transmission power needed to reach a subset of nodes and with more residual power and lower speed.

$\mathrm{In}^{[8]}$, is studied the problem of K-clustering in mobile network. K-clustering problem is NP-Complete problem to which is presented an approximation algorithm with $\theta(K)$ worst case ratio over the optimal. The idea on which is based the work presented in ${ }^{[8]}$, is to divide the network to $\mathrm{K}$ non overlapping clusters that contains all the nodes of the network. The algorithm starts the decomposition of the spanning tree from the leave to the root into sub-tree of bounded diameter. $\mathrm{In}^{[9]}$, a hierarchical clustering techniques is used to reduce the useful energy consumption where is proposed a distributed clustering algorithm HEAD (Hybrid Energy-Efficient Distributed clustering) that is based on two parameters are residual energy of each node and communication cost.

The HC is chosen given the lower intra cluster communication cost that depends on: the cluster size, the possibility of variable power transmission within the cluster (no fixed power transmission for all the nodes in the intra-cluster) and on the node degree.

Lower Id clustering is one of the most popular clustering schemes, Gerla et al. ${ }^{[12]}$ propose a simple distributed clustering algorithm in which in each cluster, exactly one node, the one with the lowest ID among its neighbors becomes a cluster head and maintains cluster membership of other member nodes. A cluster is identified by its cluster head's ID.

Some other clustering algorithms are based on the mobility models as the basis of clustering. However, the mobility is the main causes for the change in the cluster topology. In [13], is proposed MOBIC (Mobility Based Identity Clustering) algorithm hat is based on mobility metric that is used in formation of clusters which are at most 2 hops in diameter. The basic idea is that a node should not be elected a cluster head if it is highly 
mobile relative to its neighbors. Where in this situation the probability that a cluster will break and that reclustering will happen is high. For that, is selected a node that is less mobile relative to its neighbors for the role of cluster head.

$\mathrm{In}^{[14]}$, is proposed a leader election algorithm that ensures that after a finite number of topology change each node $\mathrm{i}$ has a leader which is the most valued node among all the nodes in the cluster. Where the value of a node is a performance related characteristics such as battery life, minimum average distance to the other nodes or computation capabilities.

$\mathrm{In}^{[18]}$, is proposed a leader election algorithm for wireless Ad-Hoc networks that is based on spatial information. The algorithm guarantees that exists a unique leader to each component assuming that the communication is done only by directed neighbors without any global information about the network. The system is event based where it is considered stable during one event occurrence and the system faced only one event at time. The events considered in the system are direction change, node failure, connection failure, new connection and new nods. Each event is accompanied by an election of a new leader. The time complexity of the algorithm is $\mathrm{O}\left(\mathrm{E}^{*} \mathrm{~N}\right)$ in the worst case where $\mathrm{E}$ is the number of edges and $\mathrm{N}$ is the number of nodes.

The problem affronted in this study is the leader election problem in mobile networks. Dynamic topology is the most important problem affronted in a mobile network, for that it is necessary a dynamic solution that reduce the probability of the situation where a cluster is without a leader as possible (if it happens). So, the election of a new leader must requires as less as time as possible in a manner that the topology of the network does not change significantly. For that, we propose a system in which the organization of nodes in a cluster is done given the transmission range. That means, all the nodes that are in the same cluster are within the transmission range of the root. The leader elected in our system must hold two main characteristics that are: the maximum level of power $(\mathrm{P})$ and its closeness to many nodes in the cluster (d).

Our algorithm handles the different possibilities (two leader, two leader with equal power, two leaders with the same characteristics), in addition to the mobility management. Our simulation results show that the time needed to elect a leader in our system is smaller than the time needed to a node to leave completely the area or to happen a significant change in the cluster topology. Our future work is to compare the time needed by our algorithm to elect a new leader with other related works that including adding a new parameters to our work and then studying the variation of time.

\section{REFERENCES}

1. Albini, L., A. Caruso, S. Chessa and P. Maestrini, 2004. Reliable Routing in Wireless Ad-Hoc Networks. Technical Report 2004-TR08.CNR.ISTI.PI. 3-9-2004.

2. Johnson, D.B., D.A. Maltz and Y.C. Hu, The dynamic source Routing Protocol for Mobile AdHoc Networks (DSR). IETF Internet draft, Version 9.

3. Johnson, B. and D. A.Maltz, 1996. Dynamic Source Routing in Ad-Hoc Wireless Networks. In Imielinski and Korth editors, Mobile Computing. Volume 353. Kluwer Accademic Publishers.

4. Perkins, C. and P. Bhagwat, 1994. Higly Dynamic Destination -Sequenced Distance Vector Routing (DSDV) for Mobile Computers. In ACM SIGCOMM'94. Conference on Communications Architectures Protocols and Applications, pp: 234-244.

5. Perkins, C. and E.M. Royer, 1999. Ad-Hoc on Demand Distance Vector Routing (AODV). In IEEEVMSCA 99, pp: 90-100.

6. Syrotiuk, V.R., S. Bassagni, I. Chlamatac and B.A. Woodward, 1998. A Distance Routing Effect algorithm for Mobility (DREAM). In ACM/IEEE MOBICOM 98, pp: 76-84.

7. Hwand, I.S. and W.H. Pang, 2007. Energy efficient clustering techniques for multicast routing protocol in wireless Ad-Hoc network. IJCSNS Int. J. Comput. Sci. Network Security, 7 (8).

8. Fernandess, Y. and D. Malkhi, 2002. K-clustering in wireless Ad-Hoc networks. J. Principles Mobile Comput.

9. Younis, O. and S. Fahmy, 2004. Distributed clustering in Ad-Hoc sensors networks: Hybrid energy-efficient approach. IEEE Infocom.

10. Chiang, C.C., H.K. Wu, W. Liu and Mario Gerla, 1997. Routing in Clustered Multihops, Mobile Wireless Networks with Fading Channel. Proceedings of SICON .

11. Ephremides A., J.E. Wieselthier and D.J. Baker, 1987. A design concept for reliable mobile radio networks with frequency hopping signaling . Proceedings of the IEEE, 75 (1): 56-73.

12. Gerla, M. and J.T.C. Tsa, 1995. Multicluster Mobile Multimedia Radio Networks. Wireless Networks 1. pp: 255-265. 
13. Basu, P., N. Kham and T.D.C Little, 2001. A Mobility Based Metric for Clustering in Mobile Ad-Hoc Networks. MCL Technical Report No.0115-2001. Proc IEEE ICDCS 2001 Worshop on Wireless Networks and Mobile Computing. Phoenix, AZ,Aprile 2001.

14. Vasudevan, S., J. Kurose and D. Towsley, 2004. Design and analysis of Leader Election Algorithm for Mobile Ad-Hoc Networks .

15. Malpani, N., J.L. Welch and N. Vaidya, 2000. Leader election algorithms for mobile Ad-Hoc networks. Proc Fourth International Workshop on Discrete Algorithms and Methods for Mobile Computing and Communications, pp: 96-103.
16. Park, V.D. and M.S. Corson, 1997. A Highly Adaptive Distributed Routing algorithm For Mobile Wireless Networks. Proc IEEE INFOCOM Aprile 7-11-1997.

17. Gafni, E. and D. Bertsekas, 1981. Distributed algorithms for generating loop-free route in network with frequently changing topology. IEEE Trans. Commun., C-29 (1): 11-18.

18. Lars, C.T. B. Cremean, 2004. Real Time leader election for cooperative control for dynamic vehicle formation. IEEE Real Time and Embeded Technology and Applications Symposium. 25-28 May, 2004. Toronto, Canada. 